Marcelo Souza Magalhães Bastos

Quantificação do Dano na Superfície Dentinária após Jateamento com Bicarbonato de Sódio MATERIAIS E METALURGIA

Programa de Pós-Graduação em

Engenharia Metalúrgica 


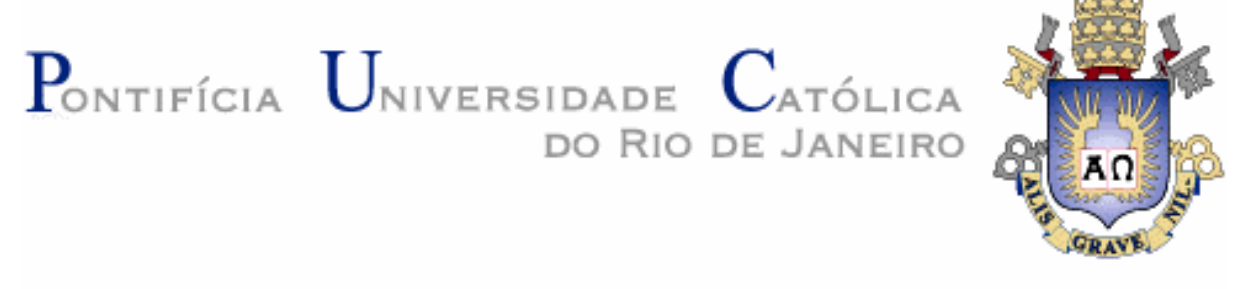

Marcelo Souza Magalhães Bastos

\section{Quantificação do Dano na Superfície Dentinária após Jateamento com Bicarbonato de Sódio}

Dissertação apresentada como requisito parcial para a obtenção do título de Mestre em Engenharia Metalúrgica e de Materiais pelo Programa de PósGraduação em Engenharia Metalúrgica do Departamento de Ciências dos Materiais e Metalurgia da PUC-Rio.

Orientador: Marcos Venicius Soares Pereira Co-Orientador: Cláudio Pinheiro Fernandes 


\title{
Marcelo Souza Magalhães Bastos
}

\section{Quantificação do Dano na Superfície Dentinária após Jateamento com Bicarbonato de Sódio}

\author{
Dissertação apresentada como requisito \\ parcial para a obtenção do grau de Mestre em \\ Engenharia Metalúrgica e de Materiais pelo \\ Programa de Pós-Graduação em Engenharia \\ Metalúrgica do Departamento de Ciência dos \\ Materiais e Metalurgia da PUC-Rio. Aprovada \\ pela Comissão Examinadora abaixo assinada.
}

Prof. Marcos Venicius Soares Pereira

Orientador

Departamento de Ciência dos Materiais e Metalurgia PUC-Rio

Prof. Cláudio Pinheiro Fernandes

Co-Orientador

Universidade Veiga de Almeida - UVA

Prof. Hélio Rodrigues Sampaio Filho Universidade do Estado do Rio de Janeiro - UERJ

Prof. Fathi Aref Ibrahim Darwish Universidade Federal Fluminense - UFF

Profa. Martha Vasconcellos Amarante Universidade Salgado de Oliveira

Prof. José Eugenio Leal Coordenador Setorial de Pós-Graduação do Centro Técnico Científico PUC-Rio 
Todos os direitos reservados. É proibida a reprodução total ou parcial do trabalho sem autorização da universidade, do autor e do orientador.

\section{Marcelo Souza Magalhães Bastos}

Dentista formado pela Faculdade de Odontologia de Campos em 1991.

Especialista em Periodontia pela Pontifícia Universidade Católica do Rio de Janeiro desde 1994.

Ficha Catalográfica

Bastos, Marcelo Souza Magalhães

Quantificação do dano na superfície dentária após jateamento com bicarbonato de sódio / Marcelo Souza Magalhães Bastos ; orientador: Marcos Venicius Soares Pereira ; co-orientador: Cláudio Fernandes. - Rio de Janeiro : PUC, Departamento de Ciência dos Materiais e Metalurgia 2005.

84 f. : il. ; $30 \mathrm{~cm}$

Dissertação (mestrado) - Pontifícia Universidade Católica do Rio de Janeiro, Departamento de Ciência dos Materiais e Metalurgia.

Inclui referências bibliográficas.

1. Ciência dos Materiais e Metalurgia - Teses. 2. Biomateriais. 3. Bioengenharia. 4. Profilaxia. 5. Microdureza. I. Pereira, Marcos Venicius Soares. II. Fernandes, Cláudio. III. Pontifícia Universidade Católica do Rio de Janeiro. Departamento de Ciência dos Materiais e Metalurgia Ciência dos Materiais e Metalurgia. IV. Título. 
Agradeço a Deus pela força e determinação que me deu para a concretização desta importante etapa em minha vida.

À minha esposa, Adriana, pelo apoio incondicional, paciência, amor e carinho, e aos meus pequeninos filhos Matheus Augusto e João Gabriel, agradeço pelo grandioso amor, e a quem peço desculpas pela minha ausência.

Aos meus pais, sementes da minha vida, grandes responsáveis pela minha educação, agradeço pelo apoio e motivação. 


\section{Agradecimentos}

A PUC-Rio por ter me proporcionado a realização deste trabalho.

Ao Prof. Marcos Venicius Soares Pereira, pelo grande apoio e orientação durante o trabalho, bem como pela oportunidade valiosa em adquirir novos conhecimentos.

Ao Prof. Cláudio Pinheiro Fernandes, pelo grande estímulo e pela colaboração concedida para utilização das dependências da Universidade Veiga de Almeida.

Ao Prof Hélio R. S. Filho da Universidade do Estado do Rio de Janeiro, pelo incentivo e colaboração oferecidos para conclusão deste trabalho.

Ao Prof. Jaime Lima da Universidade do Rio de Janeiro, por sua participação fundamental no suporte ao trabalho, como também por sua amizade.

Aos professores do mestrado e a todos os funcionários do DCMM, pela acolhida e atenção sempre dispensadas.

A todos aqueles que contribuíram direta e indiretamente para a realização deste trabalho. 


\section{Resumo}

Bastos, Marcelo Souza Magalhães. Quantificação do Dano na Superfície Dentinária após Jateamento com Bicarbonato de Sódio. Rio de Janeiro, 2005. 84f. Dissertação de Mestrado Departamento de Ciência dos Materiais e Metalurgia, Pontifícia Universidade Católica do Rio de Janeiro.

Este trabalho apresenta os resultados da quantificação do dano em superfícies dentinárias de molares humanos após jateamento com bicarbonato de sódio. Após a seleção e preparação das amostras, as mesmas foram divididas em dois grupos experimentais (Grupo Controle e Grupo de Jateamento). Inicialmente, mediu-se a rugosidade e dureza local (microdureza) do Grupo Controle, adotadas como valores padrão. Em seqüência, as amostras do Grupo de Jateamento foram submetidas à diferentes condições de jateamento, variando-se os parâmetros granulometria das partículas de bicarbonato de sódio (60 e 200 mesh), vazão da mistura água-bicarbonato de sódio-ar (mínima e máxima) e tempo de instrumentação (15 e 30 segundos). Finalmente, mediu-se a rugosidade e microdureza da região da dentina, bem como a área de depressões superficiais formada pelo jateamento. Os resultados mostraram que todas as condições de jateamento provocaram danos na região da dentina, caracterizados por aumentos de rugosidade e dureza, bem como o aparecimento de cavidades nesta região. A vazão mínima da mistura água-bicarbonato de sódio-ar provocou maiores rugosidades e endurecimentos na região dentinária. Por outro lado, as maiores áreas de cavidades na mesma região foram criadas por partículas menores quando jateadas com vazão máxima da mistura água-bicarbonato de sódio-ar.

\section{Palavras-chave}

Biomateriais; bioengenharia; profilaxia; microdureza 


\section{Abstract}

Bastos, Marcelo Souza Magalhães. Damage Quantification of Dentine Surface after Blasting with Sodium Bicarbonate. Rio de Janeiro, 2005. 84f. Master Dissertation - Department of Science of the Materials and Metallurgy, Pontifical University Catholic of Rio de Janeiro.

This work presents the results concerning the damage quantification in human molar dentine surfaces after blasting with sodium bicarbonate. After selection and preparation, the samples were divided into two experimental groups (Control Group and Blasting Group). Initially, the roughness and local hardness (microhardness) of the Control Group were measured and adopted as standard values. In the sequence, the samples of the Blasting Group were subjected to different blasting conditions, making change in parameters as grain size of the sodium bicarbonate particles (60 and 200 mesh), water-sodium bicarbonate-air mixture outflowing (minimum and maximum) and instrumentation time (15 and 30 seconds). Finally, the roughness and the microhardness of the dentine region were measured, as well as the area of the surface depressions due to blasting. The results showed that all blasting conditions caused damages in the dentine region, characterized by an increase in roughness and microhardness, as well as the creation of cavities in this region. The minimum water-sodium bicarbonate-air mixture outflowing was associated with larger values of roughness and microhardness in the dentine region. On the other hand, larger areas of cavities in the same region were created by smaller particles of sodium bicarbonate when blasted with maximum water-sodium bicarbonate-air mixture outflowing.

\section{Key words}

Biomaterials; bioengineering; prophylaxis; microhardness. 


\section{Sumário}

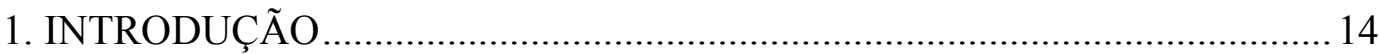

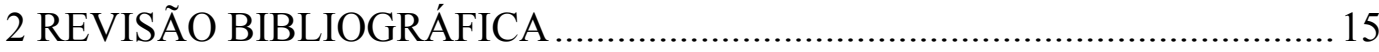

2.1 Efeito do jato de bicarbonato sobre a região de dentina coronária. 15

3 MATERIAL E PROCEDIMENTO EXPERIMENTAL ................................... 19

3.1 Seleção e Preparação das Amostras................................................ 19

3.2 Medição da Rugosidade do Grupo-Controle ................................. 20

3.3 Jateamento das Amostras................................................................ 21

3.4 Medição da Rugosidade das Amostras após Jateamento................. 25

3.5 Quantificação do Dano Causado pelo Jateamento..........................25

3.6 Medição da Microdureza das Amostras após Jateamento ...............26

4. APRESENTAÇÃO E DISCUSSÃO DOS RESULTADOS ............................. 27

4.1 Rugosidade das Amostras após Jateamento ...................................2 27

4.2 Quantificação do Dano Causado pelo Jateamento........................... 43

4.3 Microdureza das Amostras após Jateamento .................................. 59

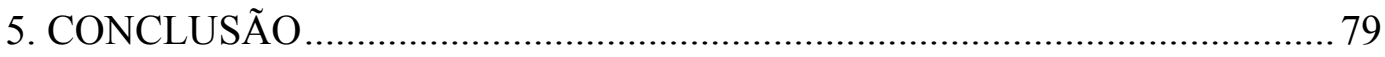

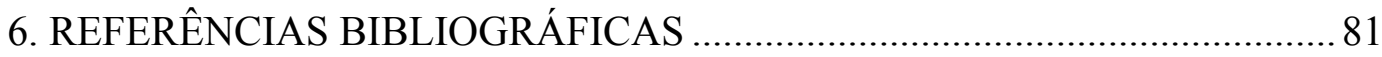




\section{Lista de Abreviaturas e Símbolos}

$\mathrm{kPa}$ - kilopascal

psi - pound per square inch (libra por polegada quadrada)

mesh - número de abertura em uma peneira por polegada linear

SPSS - Statistical Package for the Social Sciences

HV - Dureza Vickers

APD - Air-Polishing Devices (Equipamento de Polimento a Ar)

$\mathrm{NaHCO}_{3}-$ Bicarbonato de Sódio

SPT - Supportive Periodontal Therapy (Suporte da Terapia Periodontal)

$\mu \mathrm{m}$ - micrômetro 


\section{Lista de Figuras}

Figura 3.1 - Região de dentina em uma das faces das amostras

20

Figura 3.2 - Rugosímetro portátil Mitutoyo adotado nas medições de rugosidade

do Grupo-Controle.

Figura 3.3 - Dispositivo adotado no jateamento das amostras com bicarbonato

de sódio.

Figura 3.4 - Aparelho Profi II Ceramic adotado neste trabalho ........................... 23

Figura 3.5 - Jateamento de uma amostra da condição 3.................................... 24

Figura 4.1 - Amostra da condição 1 de jateamento............................................ 28

Figura 4.2 - Amostra da condição 2 de jateamento ............................................... 29

Figura 4.3 - Amostra da condição 3 de jateamento ............................................. 29

Figura 4.4 - Amostra da condição 4 de jateamento ............................................ 29

Figura 4.5 - Amostra da condição 5 de jateamento ........................................... 30

Figura 4.6 - Amostra da condição 6 de jateamento ............................................ 30

Figura 4.7 - Amostra da condição 7 de jateamento .............................................. 30

Figura 4.8 - Amostra da condição 8 de jateamento .......................................... 31

Figura 4.9 - Amostras jateadas com a mesma vazão (mínima) em tempos de 15 segundos e com mesh 60 (Grupo 1) e mesh 200 (Grupo 2).

Figura 4.10 - Amostras jateadas com mesma vazão (mínima) em tempos de 30 segundos e com mesh 60 (Grupo 3) e mesh 200 (Grupo 4)............................... 33

Figura 4.11 - Amostras jateadas com a mesma vazão (máxima) em tempos de 15 segundos e com mesh 60 (Grupo 5) e mesh 200 (Grupo 6). .............................. 34

Figura 4.12 - Amostras jateadas com a mesma vazão (máxima) em tempos de 30 segundos e com mesh 60 (Grupo 7) e mesh 200 (Grupo 8). ............................. 35

Figura 4.13 - Amostras jateadas com mesmo mesh (60) e vazão (mímina) em tempos de 15 segundos (Grupo 1) e 30 segundos (Grupo 3)............................. 36

Figura 4.14 - Amostras jateadas com mesmo mesh (200) e vazão (mímina) em tempos de 15 segundos (Grupo 2) e 30 segundos (Grupo 4)............................. 37

Figura 4.15 - Amostras jateadas com mesmo mesh (60) e vazão (máxima) em tempos de 15 segundos (Grupo 5) e 30 segundos (Grupo 7).............................. 38

Figura 4.16 - Amostras jateadas com mesmo mesh (200) e vazão (máxima) em

tempos de 15 segundos (Grupo 6) e 30 segundos (Grupo 8)............................. 39

Figura 4.17 - Amostras jateadas com mesmo mesh (60) e mesmo tempo (15s) com vazão mínima (Grupo 1) e vazão máxima (Grupo 5).

Figura 4.18 - Amostras jateadas com mesmo mesh (200) e mesmo tempo (15s)

com vazão mínima (Grupo 2) e vazão máxima (Grupo 6).

Figura 4.19 - Amostras jateadas com mesmo mesh (60) e mesmo tempo (30s) com vazão mínima (Grupo 3) e vazão máxima (Grupo 7).

Figura 4.20 - Amostras jateadas com mesmo mesh (200) e mesmo tempo (30s)

com vazão mínima (Grupo 4) e vazão máxima (Grupo 8).................................. 43

Figura 4.21 - Amostra da condição 1 de jateamento ............................................. 44

Figura 4.22 - Amostra da condição 2 de jateamento ........................................... 45

Figura 4.23 - Amostra da condição 3 de jateamento ............................................ 45

Figura 4.24 - Amostra da condição 4 de jateamento ............................................ 45 
Figura 4.25 - Amostra da condição 5 de jateamento .......................................... 46

Figura 4.26 - Amostra da condição 6 de jateamento .......................................... 46

Figura 4.27 - Amostra da condição 7 de jateamento ........................................... 46

Figura 4.28 - Amostra da condição 8 de jateamento ......................................... 47

Figura 4.29 - Amostras jateadas com a mesma vazão (mínima) em tempos de 15

segundos e com mesh 60 (Grupo 1) e mesh 200 (Grupo 2) ............................... 48

Figura 4.30 - Amostras jateadas com mesma vazão (mínima) em tempos de 30 segundos e com mesh 60 (Grupo 3) e mesh 200 (Grupo 4).

Figura 4.31 - Amostras jateadas com a mesma vazão (máxima) em tempos de 15 segundos e com mesh 60 (Grupo 5) e mesh 200 (Grupo 6) ................................ 50

Figura 4.32 - Amostras jateadas com a mesma vazão (máxima) em tempos de 30 segundos e com mesh 60 (Grupo 7) e mesh 200 (Grupo 8) ................................ 51

Figura 4.33 - Amostras jateadas com mesmo mesh (60) e vazão (mímina) em tempos de 15 segundos (Grupo 1) e 30 segundos (Grupo 3) ............................. 52 4.34 - Amostras jateadas com mesmo mesh (200) e vazão (mímina) em tempos de 15 segundos (Grupo 2) e 30 segundos (Grupo 4).

Figura 4.35 - Amostras jateadas com mesmo mesh (60) e vazão (máxima) em tempos de 15 segundos (Grupo 5) e 30 segundos (Grupo 7).............................. 54 Figura 4.36 - Amostras jateadas com mesmo mesh (200) e vazão (máxima) em tempos de 15 segundos (Grupo 6) e 30 segundos (Grupo 8).............................. 55 Figura 4.37 - Amostras jateadas com mesmo mesh (60) e mesmo tempo (15s) com vazão mínima (Grupo 1) e vazão máxima (Grupo 5)........................................... 56 Figura 4.38 - Amostras jateadas com mesmo mesh (200) e mesmo tempo (15s) com vazão mínima (Grupo 2) e vazão máxima (Grupo 6).

Figura 4.39 - Amostras jateadas com mesmo mesh (60) e mesmo tempo (30s) com vazão mínima (Grupo 3) e vazão máxima (Grupo 7)............................................ 58 Figura 4.40 - Amostras jateadas com mesmo mesh (200) e mesmo tempo (30s) com vazão mínima (Grupo 4) e vazão máxima (Grupo 8)................................... 59

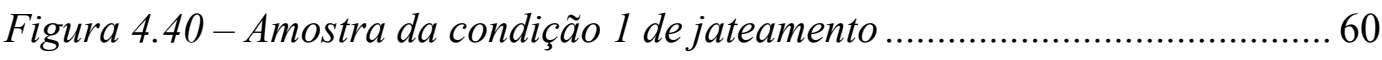

Figura 4.41 - Amostra da condição 2 de jateamento ............................................. 61

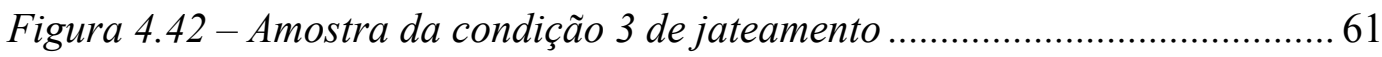

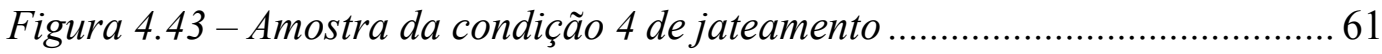

Figura 4.44 - Amostra da condição 5 de jateamento ........................................... 62

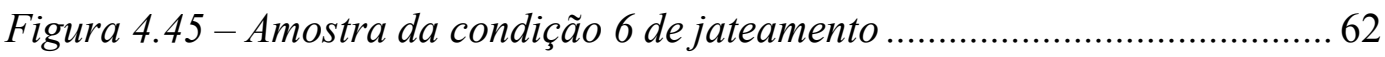

Figura 4.46 - Amostra da condição 7 de jateamento .............................................. 62

Figura 4.47 - Amostra da condição 8 de jateamento .......................................... 63

Figura 4.48 - Amostras jateadas com a mesma vazão (mínima) em tempos de 15 segundos e com mesh 60 (Grupo 1) e mesh 200 (Grupo 2). ...............................61 61

Figura 4.49 - Amostras jateadas com mesma vazão (mínima) em tempos de 30 segundos e com mesh 60 (Grupo 3) e mesh 200 (Grupo 4).

Figura 4.50 - Amostras jateadas com a mesma vazão (máxima) em tempos de 15 segundos e com mesh 60 (Grupo 5) e mesh 200 (Grupo 6). .............................. 66 Figura 4.51 - Amostras jateadas com a mesma vazão (máxima) em tempos de 30 segundos e com mesh 60 (Grupo 7) e mesh 200 (Grupo 8).

Figura 4.52 - Amostras jateadas com mesmo mesh (60) e vazão (mímina) em tempos de 15 segundos (Grupo 1) e 30 segundos (Grupo 3). 
Na condição de vazão mínima a massa referente ao grão de 60 mesh provoca um maior impacto na região a ser jateada.

Figura 4.53 - Amostras jateadas com mesmo mesh (200) e vazão (mímina) em tempos de 15 segundos (Grupo 2) e 30 segundos (Grupo 4).

Figura 4.54 - Amostras jateadas com mesmo mesh (60) e vazão (máxima) em tempos de 15 segundos (Grupo 5) e 30 segundos (Grupo 7).

Figura 4.55 - Amostras jateadas com mesmo mesh (200) e vazão (máxima) em tempos de 15 segundos (Grupo 6) e 30 segundos (Grupo 8).

Figura 4.56 - Amostras jateadas com mesmo mesh (60) e mesmo tempo (15s) com vazão mínima (Grupo 1) e vazão máxima (Grupo 5)

Figura 4.57 - Amostras jateadas com mesmo mesh (200) e mesmo tempo (15s) com vazão mínima (Grupo 2) e vazão máxima (Grupo 2).

Figura 4.58 - Amostras jateadas com mesmo mesh (60) e mesmo tempo (30s) com vazão mínima (Grupo 3) e vazão máxima (Grupo 7)

Figura 4.59 - Amostras jateadas com mesmo mesh (200) e mesmo tempo (30s) com vazão mínima (Grupo 4) e vazão máxima (Grupo 8). 


\section{Lista de Tabelas}

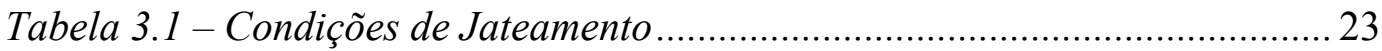

Tabela 3.2 - Composição química dos pós de bicarbonato de sódio .................... 24

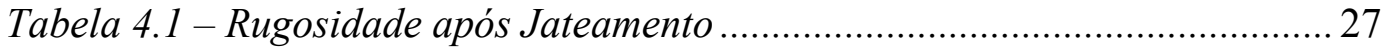

Tabela 4.2 - Área da Calota após Jateamento ....................................................... 44

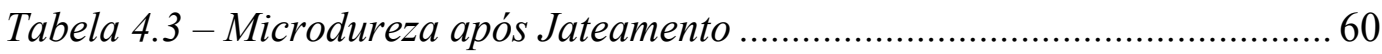

Tabela 4.4 - Características as Amostras após Jateamento................................. 76 